\title{
Design and Fabrication of Diffractive Light-Collecting Microoptical Device with $1 D$ and 2D Lamellar Grating Structures
}

\author{
ChaBum Lee \\ Department of Mechanical Engineering, University of South Carolina, 300 Main Street, Columbia, SC 29208, USA \\ Correspondence should be addressed to ChaBum Lee; chabum@mailbox.sc.edu
}

Received 6 February 2014; Accepted 17 May 2014; Published 5 June 2014

Academic Editor: Konstantinos Salonitis

Copyright (c) 2014 ChaBum Lee. This is an open access article distributed under the Creative Commons Attribution License, which permits unrestricted use, distribution, and reproduction in any medium, provided the original work is properly cited.

\begin{abstract}
This paper presents the optimal design method of diffractive light-collecting microoptical device and its fabrication method by E-beam lithography, fast atom beam etching, and hot-embossing processes. The light-collecting device proposed in the paper is comprised of $9(3 \times 3)$ blocks of optical elements: 4 blocks of $1 \mathrm{D}$ lamellar grating structures, 4 blocks of $2 \mathrm{D}$ lamellar grating structures, and a single block of nonpatterned element at the center, which acts for lens to be able to collect the diffracted and transmitted lights from the lamellar grating structures into the focus area. The overall size of the light-collecting device is $300 \times 300 \mu \mathrm{m}^{2}$, and the size of each block was practically designed as $100 \times 100 \mu \mathrm{m}^{2}$. The performance of 1D and 2D lamellar grating structures was characterized in terms of diffraction efficiency and diffraction angle using a rigorous coupled-wave analysis (RCWA) method, and those geometric parameters, depth, pitch, and orientation, were optimized to achieve a high light-collecting efficiency. The master molds for the optimized structures were fabricated on Si substrate by E-beam lithography and fast atom beam etching processes. The $100 \mu \mathrm{m}$ thick patterned polymethyl methacrylate (PMMA) film was then replicated by a hot-embossing process. As a result, the patterned PMMA film collected $63.0 \%$ more incident light than a nonpatterned one.
\end{abstract}

\section{Introduction}

Microoptical devices or hybrid integrated optical devices have been very important in the field of optical application systems such as optical communication systems, optical information processing systems, and optical sensing systems to achieve compactness and high performance [1-4]. Laser diodes (LD) and light emitting diodes (LEDs) have been widely used as light sources in these systems given their compactness, low driving current, and capability of high speed modulation [4]. The light source transmits through optical fibers in many cases; thus the output beam emitting from the source has far-field radiation angles that need an external lens such as a collimating lens or a focusing lens. But these lenses are so bulky that it is difficult to compact collimated or focus light sources for microoptical applications [3-5].

The diffractive optical elements (DOEs) can overcome such problems. Recently, a great deal of research in DOEs has been performed [6-13]. The DOEs play an important role in many optical applications, including optical telecommunications components, multiple imaging, light-collecting, and spectroscopy applications, because of their high uniformity, light weight, and miniaturization in size [1-4]. The most popular light-collecting device is a Fresnel lens as seen in Figure 1. A Fresnel lens is an optical component which can be used as a cost-effective, light-weight alternative to conventional continuous surface optics. Many fabrication methods have been proposed to produce Fresnel lenses. Fugita et al. fabricated the stepwise Fresnel lens on a Si substrate by using Ebeam lithography $[9,10]$. Yan et al. fabricated the continuous Fresnel lens on a germanium $(\mathrm{Ge})$ substrate by using ultraprecision machining [11], and Joo et al. fabricated the continuous Fresnel lens on polymeric substrate by using ultraprecision machining [12]. However, MEMS-based fabrication methods such as E-beam lithography, photolithography, and holography are limited to machining circularly curved facets and low material removal efficiency [6-10]. On the other hand, 
ultraprecision machining-based fabrication methods rely on more complicated mechanisms depending on the degree of the size effect due to small ratio of depth of cut to the tool edge radius. In addition, both MEMS-based and ultraprecision machining-based machining methods are expensive $[11,14]$. An evident disadvantage of using a lens with grooves is the possibility of losing light due to incidence on the draft facet. Making the facet perfectly vertical (i.e., perpendicular to the incident plane of light) works to minimize the optical loss (draft loss) [9-12]. However there is little work regarding thin film-type Fresnel lens for microoptics light-collecting applications.

Keeping pace with these interests in DOEs, many analysis methods have been introduced [6, 12, 13, 15-17]. Scalar diffraction theory is one of the widely used methods for the design and analysis of DOEs. The Fresnel or Fraunhofer diffraction integrals are commonly employed, and these integrals are generally calculated with the fast Fourier transform (FFT). Although this approach is relatively simple and agrees with experimental results to some extent, its applicability is limited due to diffraction limit [15-17]. When the grating size becomes so small that its scale is comparable to or less than the wavelength, the simplified approximations made in the scalar method are not valid and the polarizing nature of the light cannot be ignored in this regime. Therefore a more accurate fully electromagnetic analysis is required. The rigorous coupled-wave analysis (RCWA) provides means to solve these problems and enables characterizing the diffraction phenomenon of the DOEs with respect to TE (electric field perpendicular to the incident plane) and TM (magnetic field perpendicular to the incident plane) polarization directions.

Here thin film-type and polymethyl-methacrylate (PMMA) diffractive light-collecting microoptical device were proposed. To minimize the possibility of lost light due to incidence on the draft facet, the facet of the Fresnel lens was designed perfectly vertical. The proposed device was designed by RCWA and fabricated by E-beam lithography, fast atom beam (FAB) etching, and hot-embossing processes. The optical testing method and its performance are discussed in the following.

\section{Principles}

The configuration of the light-collecting device is presented in Figure 2, where $L$ is length of a single block, $F$ is the filling factor, $P_{1}, P_{2}$, and $P_{3}$ are periods of each block, $d$ is the depth of lens, $f_{d}$ is the focal length, and $n_{1}$ and $n_{0}$ are refractive index of PMMA and air. It is comprised of $9(3 \times 3)$ blocks of optical elements: 4 blocks of 1D lamellar grating structures, 4 blocks of 2D lamellar grating structures, and a single block of nonpatterned element at the center, which acts for lens to be able to collect the diffractive and transmitted lights from the lamellar grating structures into the center of the structure. The overall size of the light-collecting device is 300 $\times 300 \mu \mathrm{m}^{2}$, and the size of each block was practically designed as $100 \times 100 \mu \mathrm{m}^{2}$. As seen in Figure 2(b), the incident light is diffracted from 8 blocks of $1 \mathrm{D}$ and $2 \mathrm{D}$ lamellar grating
TABLE 1: Optical and geometrical conditions of 1D and 2D DOEs for optical analysis.

\begin{tabular}{lccccc}
\hline Type & $\lambda[\mu \mathrm{m}]$ & $n_{1} / n_{0}$ & $P[\mu \mathrm{m}]$ & $F$ & $D[\mu \mathrm{m}]$ \\
\hline $1 \mathrm{D}$ & 0.65 & 1.49 & $\begin{array}{c}2.0 \\
1.428\end{array}$ & 0.5 & 0.65 \\
$2 \mathrm{D}$ & & & & \\
\hline
\end{tabular}

structures, and each diffracted light will be focused on a projected nonpatterned area with $f_{d}$.

The pitches of $1 \mathrm{D}$ and $2 \mathrm{D}$ grating structures were determined by [5]

$$
n_{0} \sin \left(\theta_{0}\right)+n_{1} \sin \left(\theta_{1}\right)=m \frac{\lambda}{P}
$$

where $m$ is the number of diffraction order. The pitches of $1 D$ grating structures, $P_{2}$ and $P_{3}$, were practically set to $2.0 \mu \mathrm{m}$, which corresponded to diffraction angle, $\pm 12.6^{\circ}$ in degree. In accordance with the pitch of $1 \mathrm{D}$ grating structures, the pitch of $2 \mathrm{D}$ grating structures was determined to make the incident light diffract into the center area, which was $\pm 17.7^{\circ}$ in degree. The phase-matched depth, $d$, was optimized using RCWA under the optical and geometrical properties as summarized in Table 1. Diffraction efficiencies were calculated by RCWA with respect to the grating depth as seen in Figure 3. The 0 th order diffraction efficiencies reached their minimum at depths of $0.63 \mu \mathrm{m}$ and $0.66 \mu \mathrm{m}$ for TE and TM waves, respectively, while the \pm 1 st order diffraction efficiencies reached their maximum at these depths. The \pm 1 st order diffraction efficiencies with respect to polarization directions, $\eta_{1, \mathrm{TE}}$ and $\eta_{1, \mathrm{TM}}$, were estimated to be $38.3 \%$ and $38.7 \%$, at a depth of $0.63 \mu \mathrm{m}$ and $0.66 \mu \mathrm{m}$, respectively. Although small difference existed between diffraction efficiencies with respect to TE and TM waves, it is small enough that the depth was set to $0.65 \mu \mathrm{m}$. Diffraction angles were calculated to confirm the estimated angles using the fast Fourier transform as seen in Figure 4, which showed good agreement with the estimated ones. From the pitches of grating structures, the focal length was calculated, $448 \mu \mathrm{m}$.

\section{Fabrication}

The diffractive light-collecting microoptical device as a mold master was fabricated on a Si substrate by E-beam lithography (ELS 3700, Elionix) and FAB (FAB 60ML, Abara) processes, and the PMMA replica was duplicated by hot-embossing process. E-beam lithography and FAB processes were shown in Figure 5. An n-type of $500 \mu \mathrm{m}$ thick Si substrate $(20 \mathrm{~mm}$ by $20 \mathrm{~mm}$ ) was used for E-beam direct writing. For the fabrication, $1000 \mathrm{~nm}$ thick PR (photoresistor, ZEP 520, Nippon Zeon Co., Ltd.) was coated on the silicon substrate and prebaked at 180 degree for $3 \mathrm{~min}$ as seen in Figure 5(b). After that, E-beam direct writing was used to fabricate the PR patterned $1 \mathrm{D}$ and 2D DOEs; the acceleration voltage, beam current, and dose time were $30 \mathrm{KV}, 10 \mathrm{pA}$, and $35 \mu \mathrm{s}$, respectively, which corresponds to a dose of $56 \mu \mathrm{C} / \mathrm{cm}^{2}$. Next, E-beam exposed silicon substrate was developed in ZEP 520 developer for $5 \mathrm{~min}$ and washed in the rinse-filled beaker (methyl isobutyl ketone) for $30 \mathrm{sec}$ in the water pool at 


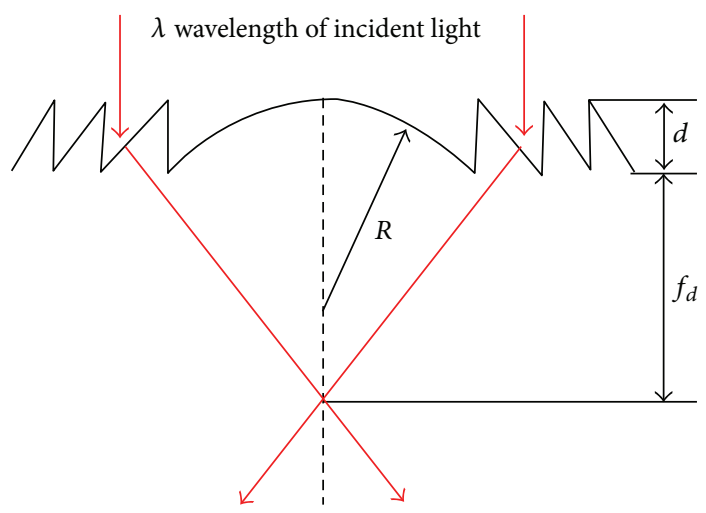

FIGURE 1: Schematics of general Fresnel lens: $d$ depth of lens, $f_{d}$ focal length, and $R$ radius of curvature.

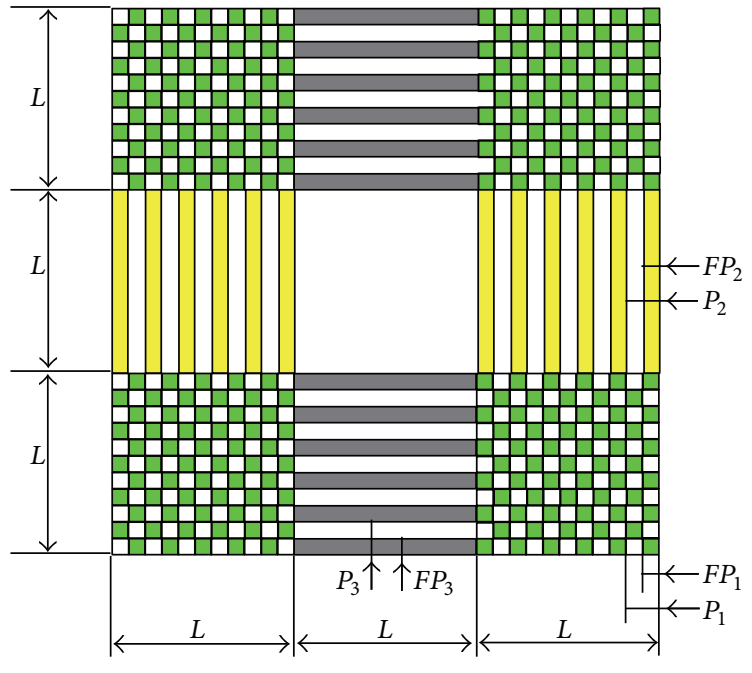

(a)

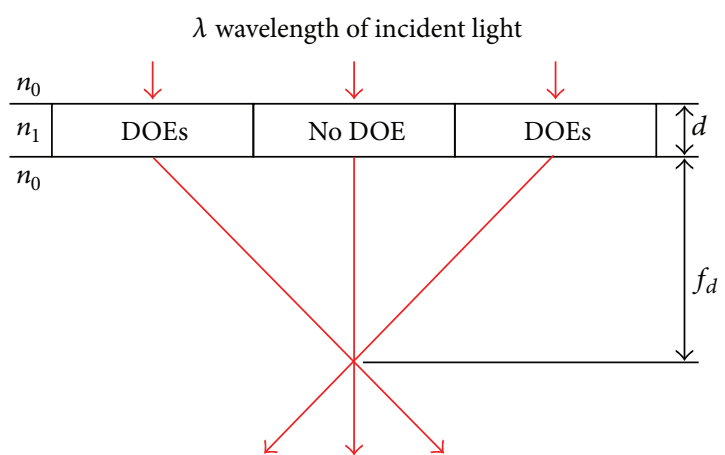

(b)

FIGURE 2: Schematics of the proposed light-collecting device: (a) overall layout: $9(3 \times 3)$ blocks of optical elements: 4 blocks of $1 D$ lamellar grating structures, 4 blocks of 2D lamellar grating structures, and a single block of nonpatterned element at the center and (b) cross-sectional view: $d$ depth of lens and $f_{d}$ focal length.

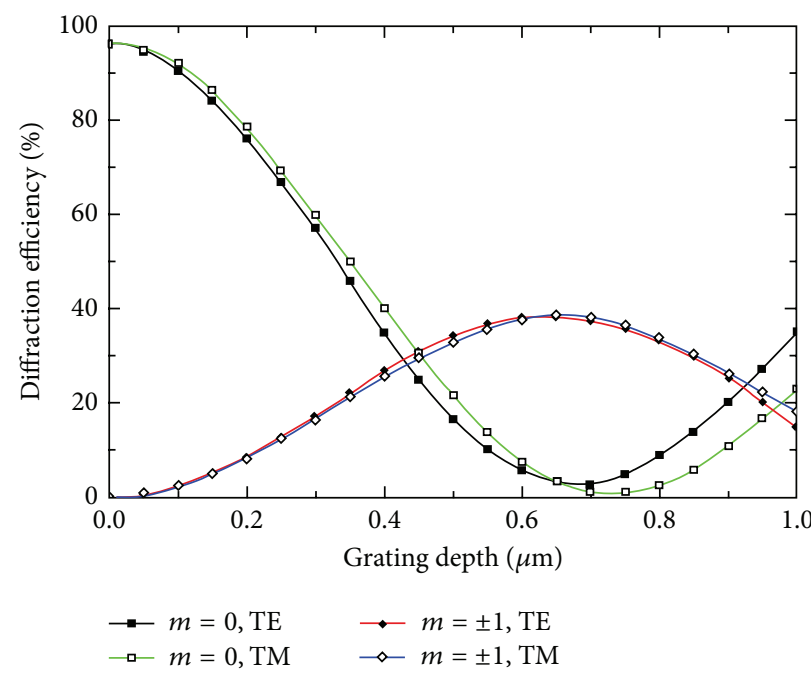

FIgure 3: Diffraction efficiency curve with respect to the grating depth. 


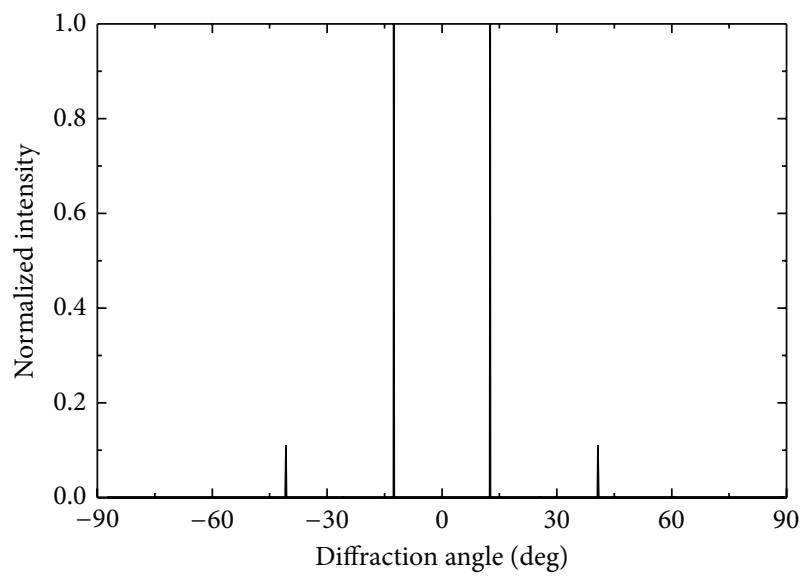

(a)

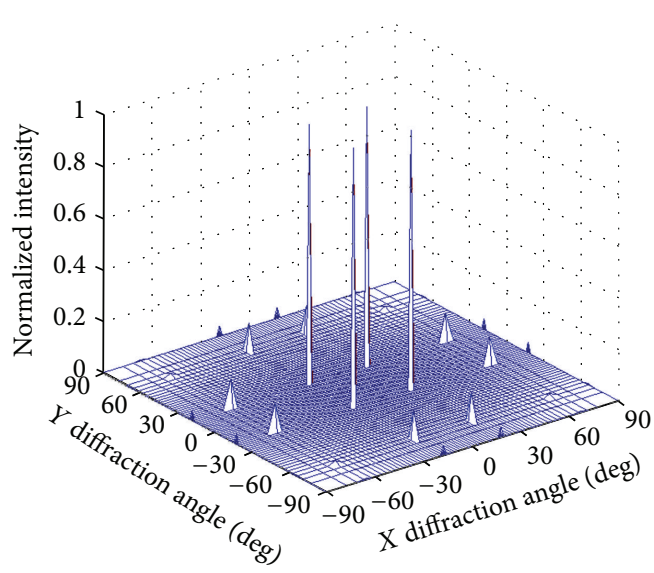

(b)

Figure 4: The estimated diffraction angles of $1 \mathrm{D}$ and $2 \mathrm{D}$ DOEs; (a) $\theta \pm 1$ st $= \pm 12.6^{\circ}$ for $1 \mathrm{D}$, (b) $\theta \pm 1$ st $= \pm 17.7^{\circ}$ for $2 \mathrm{D}$.

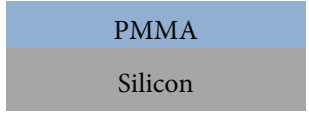

(a)

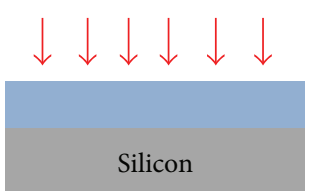

(c)

(b)

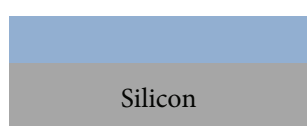

(d)

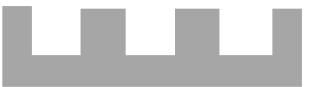

(e)

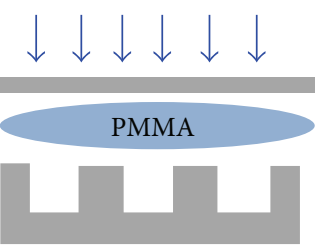

(f)

FIGURE 5: Fabrication process of DOEs; (a) silicon substrate cleaning, (b) PR coating, (c) E-beam lithography, (d) developing, (e) FAB etching, and (f) hot-embossing molding.

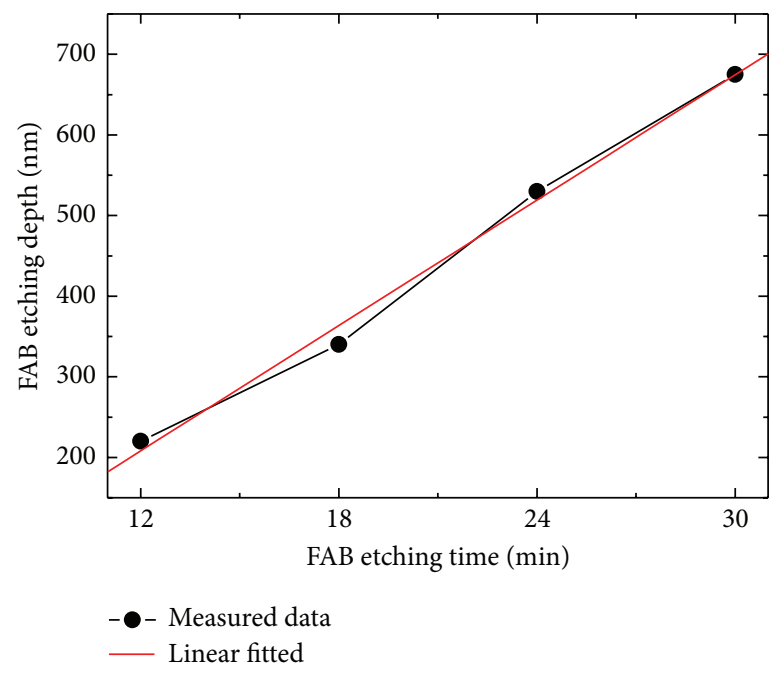

FIGURE 6: Calibration of FAB etching ratio: $21.0 \mathrm{~nm} / \mathrm{min}$. 


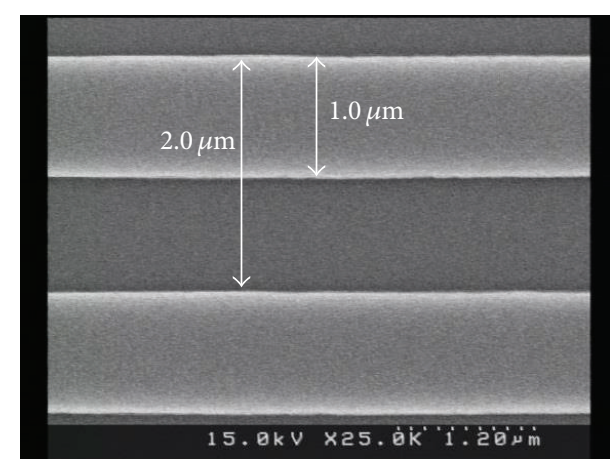

(a)

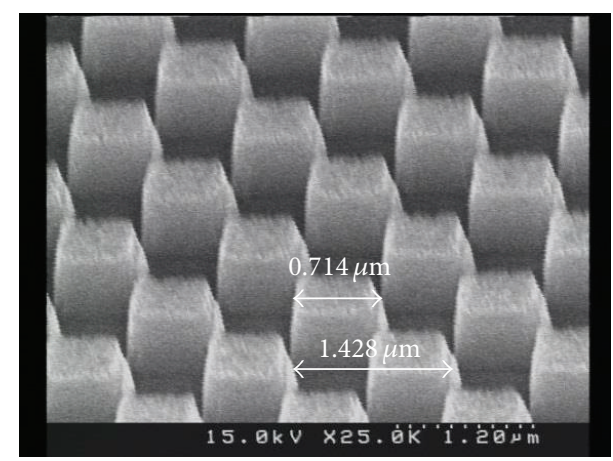

(c)

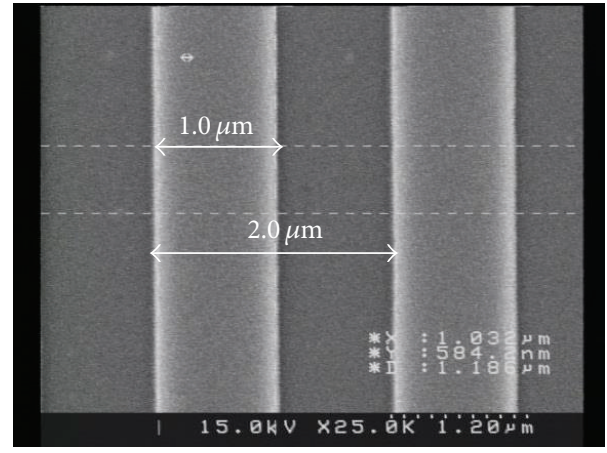

(b)

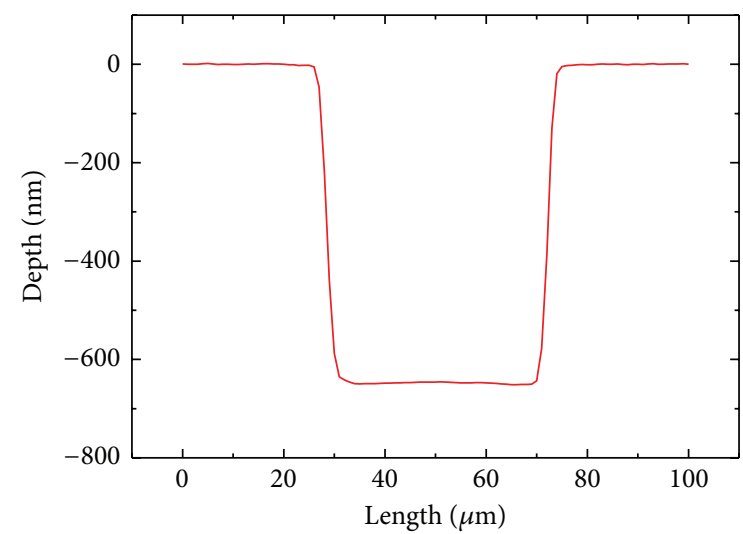

(d)

FIGURE 7: Scanning electron micrograph of the fabricated 1D and 2D DOEs and the depth measured by surface profiler; (a) horizontal 1D DOEs (yellow structures in Figure 2), (b) vertical ID DOEs (gray structures in Figure 2), (c) 2D DOEs, and (d) the depth of each DOE.

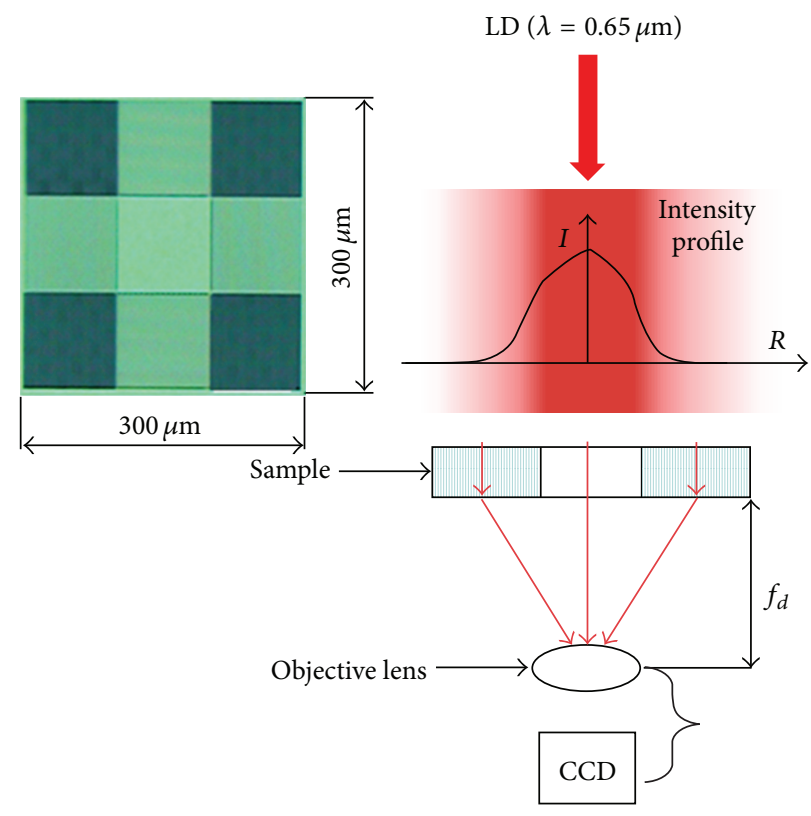

Figure 8: Experimental setup for focal length measurement. 


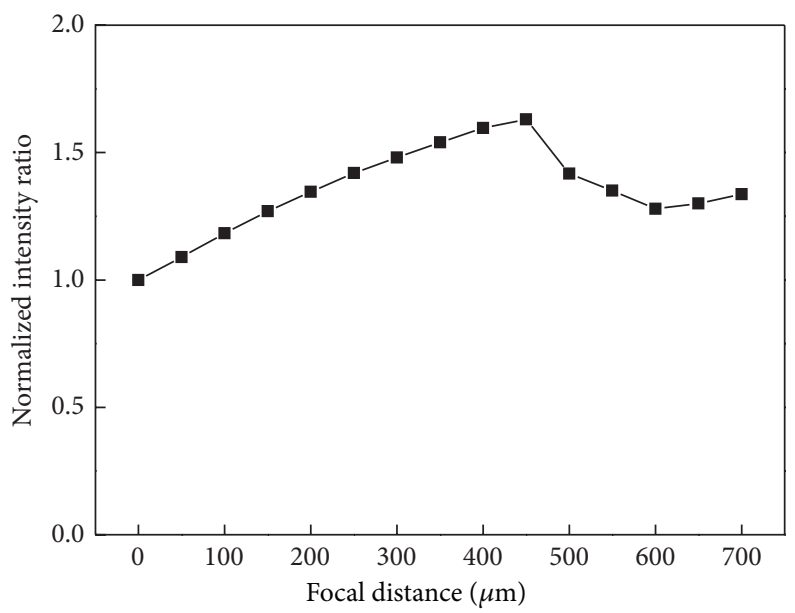

FIgURE 9: Measurement result of the intensity collected at each focal distance.

a temperature of $23^{\circ} \mathrm{C}$ and then postbaked for $3 \mathrm{~min}$ at $110^{\circ} \mathrm{C}$. As seen in Figure 5(d), the PR patterned 1D and 2D DOEs $(300 \mu \mathrm{m}$ by $300 \mu \mathrm{m})$ on the silicon substrate were fabricated. FAB etching has a good directionality and a constant etching ratio $(1: 1)$ between the silicon and $\mathrm{PR}[10,13,18,19]$. The FAB etching ratio, $21.0 \mathrm{~nm} / \mathrm{min}$, was calibrated under an etching condition as shown in Figure 6. The DOEs with a depth of $0.65 \mu \mathrm{m}$ and a period of $2.0 \mu \mathrm{m}$ and $1.43 \mu \mathrm{m}$ were successfully fabricated in Figure 5(e) and both fabricated 1D and 2D DOEs were taken by scanning electron microscope and etched depth was measured by surface profiler (Tencor). These results were shown in Figure 7. The etched depth of Si substrate was approximately $0.65 \mu \mathrm{m}$ the same as design value. Last, the patterned Si substrate was hydrophobically coated to make the mold release easier. The $100 \mu \mathrm{m}$ thick PMMA patterned film was replicated from the patterned $\mathrm{Si}$ substrate as a master mold by hot-embossing operation. The optimal hot-embossing condition was investigated by its process sequence: preheating, pressing, cooling, and demolding $[10,12,13]$. The hot-embossing condition was set: molding temperature $150^{\circ} \mathrm{C}$, applied pressure $0.6 \mathrm{MPa}$, pressing time $5 \mathrm{~min}$, and demolding temperature $25^{\circ} \mathrm{C}$.

\section{Results}

The measurement setup was shown in Figure 8. The LD ( $\lambda=0.65 \mu \mathrm{m}$, Neoark) was used as the light source with a Gaussian intensity distribution, and the optical fiber was used to deliver the light from the LD. The fabricated device was placed after the optical fiber, and the light-collecting result was measured by the CCD while moving the objective lens upward and downward manually. The digital dial gauge was used to measure the moving height. The measurement results were observed at every $50 \mu \mathrm{m}$ from 0 to $700 \mu \mathrm{m}$ and these images were photo-taken by CCD. The intensities of each image were estimated by gray scale, and the normalized intensity ratio was calculated with respect to focal distances as shown in Figure 9. As a result, the maximum intensity was measured at a focal distance with $450 \mu \mathrm{m}$ and increased by $63.0 \%$ compared to the intensity of nonpatterned area only, which has good agreement with estimated focal length, $448 \mu \mathrm{m}$.

\section{Conclusion}

The new microoptical device for the purpose of the lightcollecting was proposed and fabricated. Grating structures were optimized by RCWA and fabricated by E-beam lithography and fast atom beam etching processes. The mold master fabricated on $\mathrm{Si}$ substrate was replicated into thin filmtype $(100 \mu \mathrm{m})$ PMMA replica by hot embossing. From the measurement results, the device was successfully fabricated, and the shape of grating structures, pitch, filling factor, and depth were the same as designed. The maximum intensity was measured at a focal distance of $450 \mu \mathrm{m}$ and increased by $63.0 \%$ compared to the intensity of nonpatterned area only, which has good agreement with estimated focal length, $448 \mu \mathrm{m}$. As a result, the proposed light-collecting lens system showed high performance including high uniformity, light weight, and miniaturization in size for the purpose of the LD light focusing applications. This design approach is expected to be applied to various microoptical applications such as color mixing/filtering, light collimation, and light focusing areas.

\section{Conflict of Interests}

The author declares that there is no conflict of interests regarding the publication of this paper.

\section{References}

[1] N. F. Borrelli, Microoptics Technology, Marcel Dekker, 2nd edition, 2004.

[2] G. I. Hatakoshi and S. I. Tanaka, "Grating lenses for integrated optics," Optics Letters, vol. 2, no. 6, pp. 142-144, 1978.

[3] J. Y. Joo, D.-K. Woo, S. S. Park, and S.-K. Lee, "Design and fabrication of a fingerprint imager with compact LED illumination 
and compact imaging optics," Optics Express, vol. 18, no. 18, pp. 18932-18944, 2010.

[4] H. Goto, Y. Ito, H. Sekii, S. Ogata, and K. Imanaka, "Micro collimated light source using micro Fresnel lens," in Micro System Technologies 90, pp. 471-476, 1990.

[5] J. W. Goodman, Introduction to Fourier Optics, McGraw-Hill, San Francisco, Calif, USA, 1968.

[6] I. Kallioniemi, T. Ammer, and M. Rossi, "Optimization of continuous-profile blazed gratings using rigorous diffraction theory," Optics Communications, vol. 177, no. 1, pp. 15-24, 2000.

[7] C. David, J. Wei, T. Lippert, and A. Wokaun, "Diffractive greytone phase masks for laser ablation lithography," Microelectronic Engineering, vol. 57-58, pp. 453-460, 2001.

[8] M. Okano, H. Kikuta, Y. Hirai, K. Yamamoto, and T. Yotsuya, "Optimization of diffraction grating profiles in fabrication by electron-beam lithography," Applied Optics, vol. 43, no. 27, pp. 5137-5142, 2004.

[9] T. Fugita, H. Nishihara, and J. Koyama, "Blazed gratings and Fresnel lenses fabricated by electron-beam lithography," Optics Letters, vol. 7, no. 12, pp. 578-580, 1982.

[10] D.-K. Woo, K. Hane, and S.-K. Lee, "Fabrication of a multi-level lens using independent-exposure lithography and FAB plasma etching," Journal of Optics A: Pure and Applied Optics, vol. 10, no. 4, Article ID 044001, 2008.

[11] J. Yan, K. Maekawa, J. Tamaki, and T. Kuriyagawa, "Micro grooving on single-crystal germanium for infrared Fresnel lenses," Journal of Micromechanics and Microengineering, vol. 15, no. 10, pp. 1925-1931, 2005.

[12] J. Y. Joo, C. S. Kang, S. S. Park, and S.-K. Lee, "LED beam shaping lens based on the near-field illumination," Optics Express, vol. 17, no. 26, pp. 23449-23458, 2009.

[13] C. B. Lee, T. Kuriyagawa, D.-K. Woo, and S.-K. Lee, “Optimizing the fabrication process of a high-efficiency blazed grating through diamond scribing and molding," Journal of Micromechanics and Microengineering, vol. 20, no. 5, Article ID 055028, 2010.

[14] C. B. Lee, K. Hane, and S.-K. Lee, “The optimization of sawtooth gratings using RCWA and its fabrication on a slanted silicon substrate by fast atom beam etching," Journal of Micromechanics and Microengineering, vol. 18, no. 4, Article ID 045014, 2008.

[15] D. Dornfeld, S. Min, and Y. Takeuchi, "Recent advances in mechanical micromachining," CIRP Annals-Manufacturing Technology, vol. 55, no. 2, pp. 745-768, 2006.

[16] M. G. Moharam and T. K. Gaylord, "Rigorous coupled-wave analysis of planar-grating diffraction," Journal of the Optical Society of America, vol. 71, no. 7, pp. 811-818, 1981.

[17] M. G. Moharam, D. A. Pommet, E. B. Grann, and T. K. Gaylord, "Stable implementation of the rigorous coupled-wave analysis for surface-relief gratings: enhanced transmittance matrix approach," Journal of the Optical Society of America A, vol. 12, no. 5, pp. 1077-1086, 1995.

[18] M. G. Moharam and T. K. Gaylord, "Diffraction analysis of dielectric surface-relief gratings," Journal of the Optical Society of America, vol. 72, no. 10, pp. 1385-1392, 1982.

[19] F. Shimokawa and H. Kuwano, "New high-power fast atom beam source," Journal of Vacuum Science \& Technology A, vol. 12, no. 5, pp. 2739-2744, 1994. 

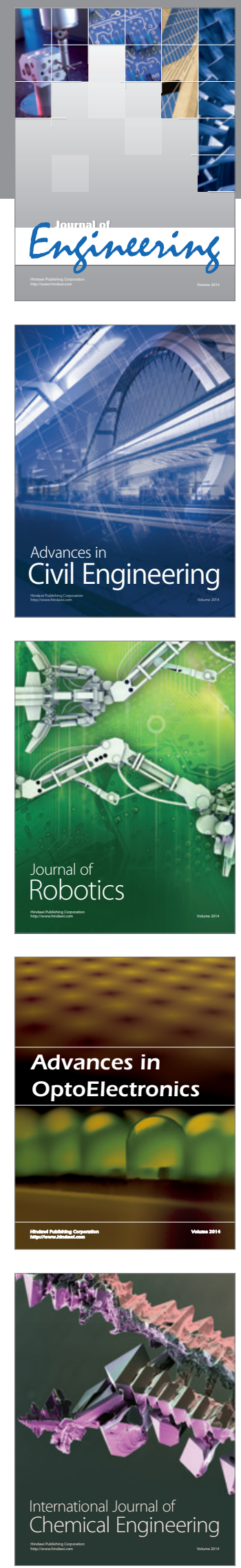

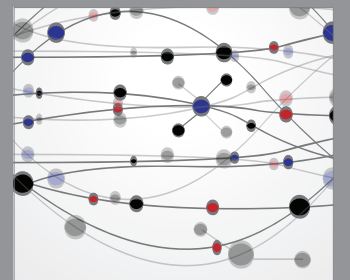

The Scientific World Journal
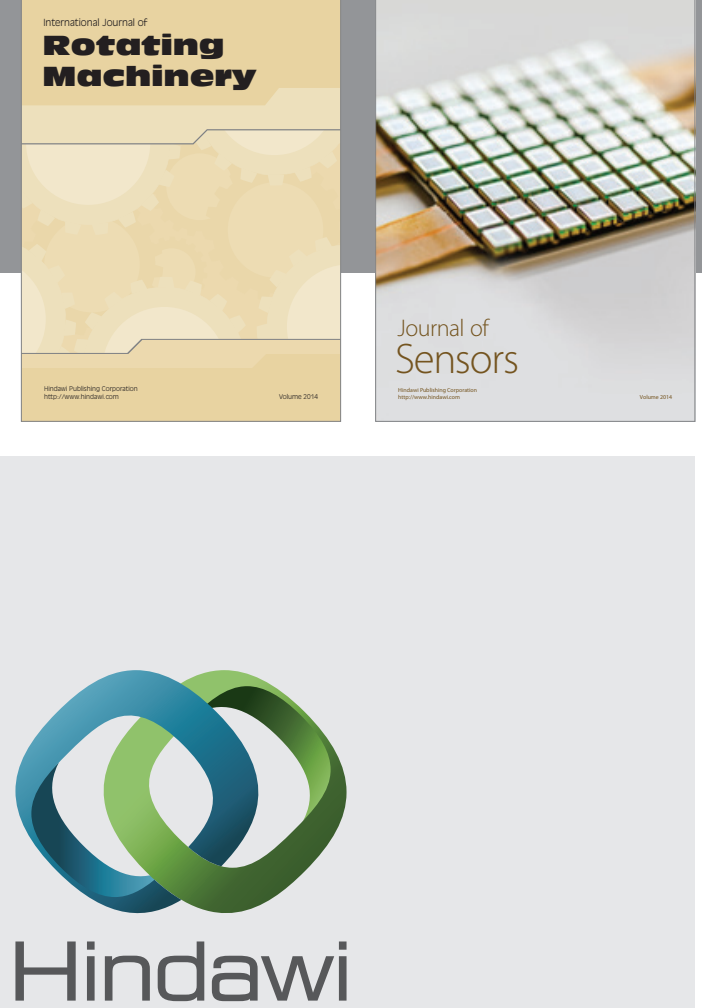

Submit your manuscripts at http://www.hindawi.com
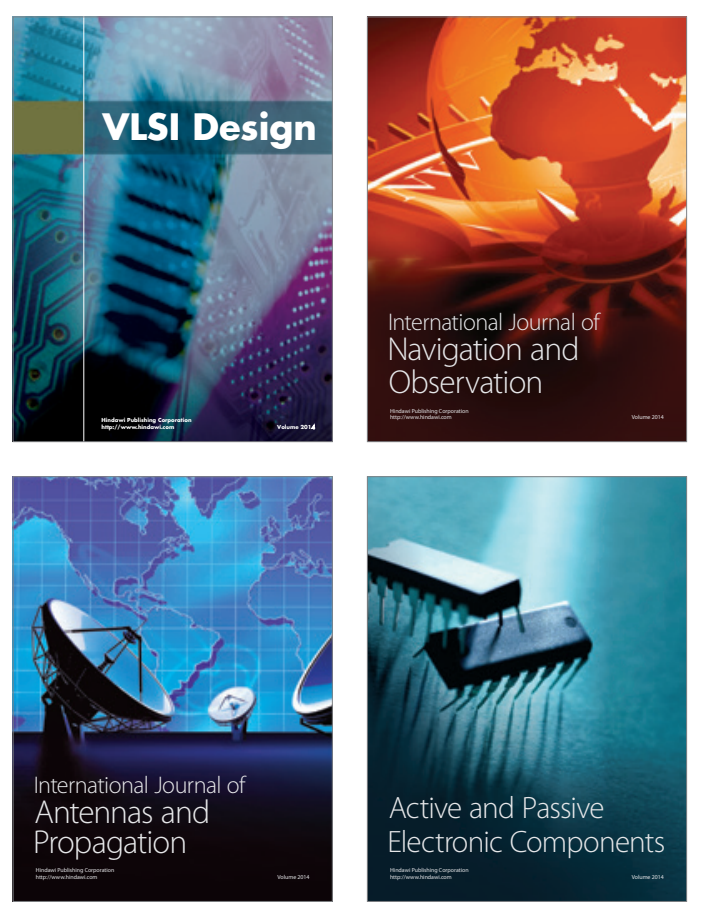
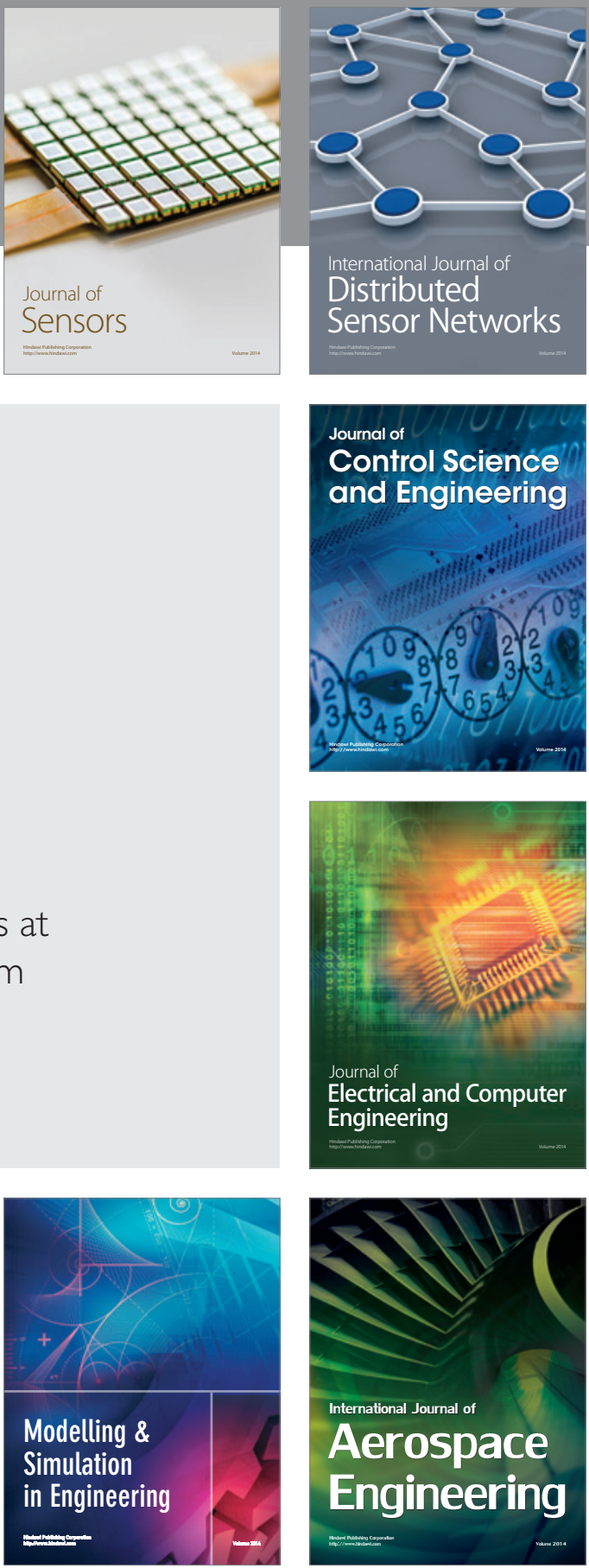

Journal of

Control Science

and Engineering
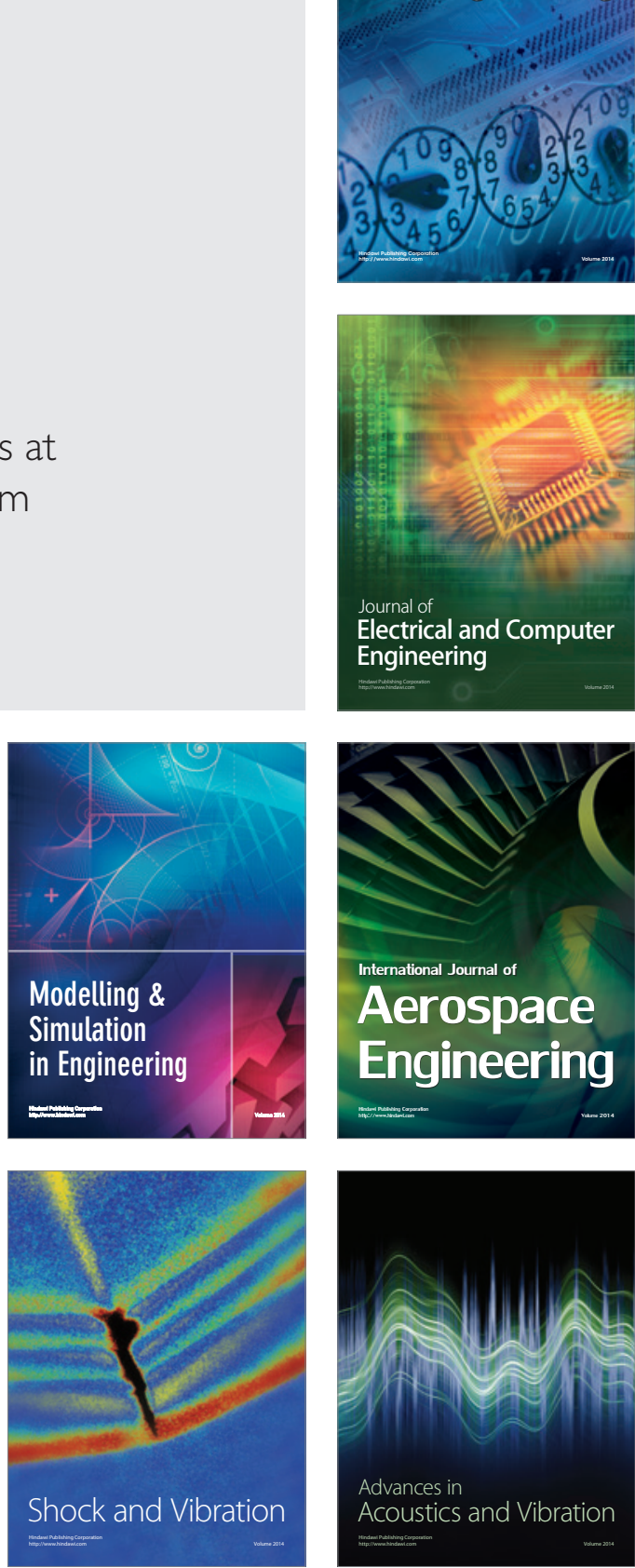